\title{
Effects of Assist-as-needed Robotic Training Paradigms on the Locomotor Recovery of Adult Spinal Mice
}

\author{
Lance L. Cai ${ }^{1}$, Andy J. Fong ${ }^{1}$, Yongqiang Liang ${ }^{2}$ \\ and Joel Burdick ${ }^{2}$ \\ 1. Bioengineering and ${ }^{2 .}$ Mechanical Engineering \\ California Institute of Technology \\ 1200 E. California Blvd. \\ Pasadena, CA 91125, USA
}

\author{
Chad K. Otoshi ${ }^{1}$ and V. Reggie Edgerton ${ }^{2,3}$ \\ Dept. ${ }^{1 .}$ Neurobiology, ${ }^{2}$ Physiological Science \\ and ${ }^{3}$ Brain Research Institute \\ University of California, Los Angeles \\ 621 Charles E. Young Dr. South \\ Los Angeles, CA 90095, USA
}

\begin{abstract}
This paper introduces a new "assist-asneeded" (AAN) training paradigm for rehabilitation of spinal cord injuries via robotic training devices. In the pilot study reported in this paper, nine female adult Swiss-Webster mice were divided into three groups, each experiencing a different robotic training control strategy: a fixed training trajectory (Fixed Group, A), an AAN training method without interlimb coordination (Band Group, B), and an AAN training method with bilateral hindlimb coordination (Window Group, C). Fourteen days after complete transection at the mid-thoracic level, the mice were robotically trained to step in the presence of an acutely administered serotonin agonist, quipazine, for a period of six weeks. The mice that received AAN training (Groups B and C) show higher levels of recovery than Group A mice, as measured by the number, consistency, and periodicity of steps realized during testing sessions. Group $\mathrm{C}$ displays a higher incidence of alternating stepping than Group B. These results indicate that this training approach may be more effective than fixed trajectory paradigms in promoting robust post-injury stepping behavior. Furthermore, the constraint of interlimb coordination appears to be an important contribution to successful training. Presented in this paper are also some preliminary results from a recent full-scale study that complements the conclusions from this pilot study.
\end{abstract}

\section{INTRODUCTION}

Robotically facilitated rehabilitative training paradigms have recently become more common. Commercially available robotic orthotics such as the Lokomat $\mathrm{TM}$ are already available to facilitate the rehabilitative training of spinal cord injured (SCI) and stroke patients with promising results [1]. Additionally, robotic devices are increasingly used for studies on animal models of spinal cord

This work is supported by the Christopher Reeve Paralysis Foundation (\#VEC 2002-2), the National Institutes of Child Health and Humane Development and the National Institute of Neural Disorders and Stroke (1 R01 HD044830-01). injuries [2, 3]. Moreover, a growing body of literature suggests that robotic systems and mechanical linkages can play an important role in post-stroke recovery of arm function [4].

However, little is known about the physiological mechanisms of how locomotor recovery is achieved with this type of rehabilitative training. Additionally the best training procedure has yet to be established.

The long term goals of our work are to:

- develop an understanding of the neurophysiological mechanisms underlying locomotor recovery;

- develop the technology and drug therapies to facilitate rapid and optimal recovery;

- develop the best training procedures that lead to rapid and sustained recovery.

Clearly, robotic devices can play an important role in the recovery process. They can provide cost effective therapy (by augmenting the capabilities of human physical therapists), they can quantitatively measure and monitor the key physiological variables related to recovery (thereby giving quantitative assessment of recovery progress), and they can potentially provide adaptive therapies tailored to each patient. Robotic devices can also help automate and systematize the process of spinal cord injury research.

In this paper, we use a robotic training device (Fig. 1) coupled with administration of quipazine (a serotonin agonist) to study locomotor recovery after SCI in mice. Our use of quipazine is motivated by the fact that there are significant biochemical changes in the spinal cord caudal to the lesion post SCI. The serotonin system is one of the principal neurotransmitter systems, responsible for regulating a diverse array of behaviors. Since its primary production is in the raphe nucleus in the brain, post SCI the level of serotonin decrease drastically in the spinal cord caudal to the lesion. It has been previously observed that pharmacological treatment with the drug quipazine can facilitate stepping in animals whose spinal cords have been completely severed [5-7]. In addition, we have shown previously that the level of recovery is greater when robotic training is combine with the admini- 




Figure 1: Schematic of current step training system. Important components are labeled: A) Optical encoder; B) Motor; C) Weight support; D) Manipulators; and E) Motorized treadmill.

stration of quipazine [8]. However, the underlying mechanism of how quipazine facilitate locomotion is still unknown. Thus a mouse model is attractive due to the many strains of transgenic mice available to study detailed issues in the role of pharmacology, such as quipazine, in locomotion recovery after SCI.

To date, the algorithms that have been used in robotic locomotion training devices have almost exclusively focused on repeated movements of the limbs through fixed kinematic trajectories. However, fixed, repetitive training is very likely to be suboptimal, as variability is an intrinsic property of mammalian locomotion [5]. Hence, fixed locomotor training may lead to the phenomena of "learned helplessness" [6, 7]. That is, the lower spinal cord becomes habituated to the training trajectory. Thus, we believe that fixed trajectory training will eventually cause an extensive level of habituation to sensory inputs such that there is eventually little or no response to the sensory inputs imposed by the robotic training device and, therefore, little or no motor output is generated. As a consequence, the training becomes counter productive, causing the rate of rehabilitation to decrease, and preventing the locomotion control circuits from robustly handling stepping trajectories other than the trained pattern [8].

In this paper, we propose and test the hypothesis that the post-SCI spinal cord will be better able to relearn to step if it is constantly challenged during locomotor training by introducing flexibility in the training pattern. We implement this in the form of an "assist-asneeded" (AAN) training paradigm. Our AAN algorithms provide strong feedback guidance when the subject's stepping pattern is far from the nominal pattern. However, only a gentle form of guidance is provided when the animal is close to the desired trajectory, thereby allowing the animal to largely guide its own motions when it is doing well. In this way, a reasonable amount of variability in the stepping trajectory is experienced during training, similar to normal locomotion. We hypothesize that this form of guidance leads to better and more robust spinal cord learning and locomotion recovery.

There are many possible variations on the AAN theme. The present pilot study compared the efficacy of two AAN robotic training algorithms versus fixed trajectory robotic training on recovery of locomotory ability in completely spinalized adult mice that were administered quipazine. The results of the study show that mice undergoing AAN robotic training exhibited faster and more pronounced recovery than mice given fixed robotic training. These findings suggest that an improved rehabilitation strategy can be realized by combining an "assist as needed" training paradigm and pharmacological intervention. The exact optimal AAN algorithm still needs to be developed. However, the results presented in this paper show the benefits of the AAN paradigm, and suggest avenues for future refinement.

This paper is structured as follows. Section II describes our experimental methods and apparatus, while Section III details the fixed and AAN training algorithms. Section IV describes the techniques used to evaluate locomotion recovery. Section V summarizes the experimental results, while Section VI discusses our results.

\section{EXPERIMENTAL METHODS AND APPARATUS}

Animals: Our experiment used adult Swiss-Webster mice (mean body weight of $21.5 \pm 1.1 \mathrm{~g}$ at the time of surgery) obtained from Charles River Laboratories (Wilmington, MA) were used. A complete mid-thoracic spinal transection was performed at 45 days of age.

Quipazine Administration: Quipazine $(0.5 \mu \mathrm{g} / \mathrm{g}$ body weight, i.p.) was administered to all subjects 5 min prior to each training or testing session [9].

Robotic Step-Training and Measurement System: A four axis robotic system was developed for both active training and data acquisition (see Fig. 1 for a schematic)

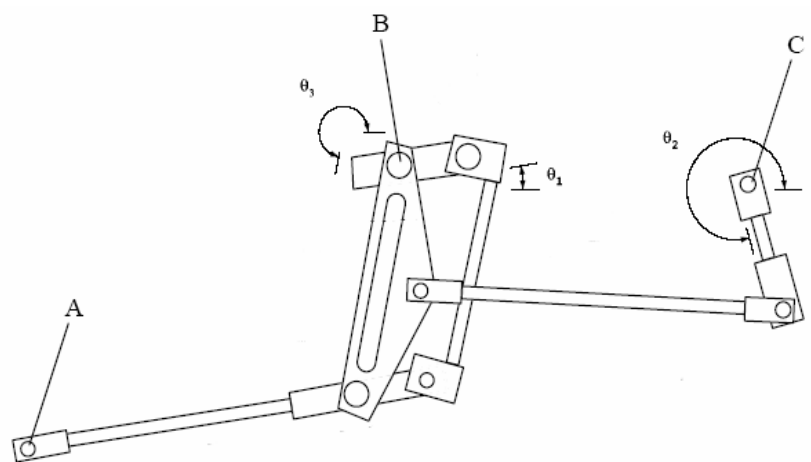

Figure 2: Schematic diagram (drawn to scale) of the 5-bar, parallelogram-type linkage. Labeled features are: A) ankle attachment point; B) motor attachment point driving linkage 4; C) motor attachment point driving linkage 1 . 
of mouse limb movements. The robotic system consists of four major components: 1) a pair of 2 DOF robotic manipulators to guide and measure ankle motions in the sagittal plane; 2) motion control hardware; 3) a treadmill; and 4) a body weight-support device.

Each 2 DOF robotic manipulator is comprised of a 5-bar leg guidance linkage (Fig. 2), a pair of motors that drive the linkage, and a pair of optical encoders to record the motors' rotational position. Ankle position is derived from these encoders and the linkage geometry. The five bar limb guidance linkage allows motion sensing and control in a vertical (sagittal) $3.5 \times 3.5 \mathrm{~cm}$ workspace plane, which is sufficient to accommodate all step trajectories associated with mouse locomotion training. In their active mode, the robotic arms drive the two hindlimbs. In their passive mode, the linkages move freely in the workspace while attached to the ankles, allowing independent measurements of the ankle movements generated by hindlimbs of the mouse.

Due to the linkage geometry, the dynamics of the two axes are approximately uncoupled and approximately linear in the range of motions encountered during training and evaluation. To minimize encumbrance on the limbs of the mice during passive operation, precision bearings and motors with low internal friction were used at all revolute joints. We estimate the frictional resistance force at the end-effector to be $0.032 \mathrm{~N}$. We estimate the mass inertia of the robotic arm linkage, including its actuators, to be $\sim 0.4 \mathrm{~g}$ in its home configuration. Although mass inertia varies with orientation, the maximum mass inertia felt by the mouse is on the same order of magnitude as the home configuration value, and hence is sufficiently small to allow us to assert that the robotic arms do not critically hinder stepping.

The mice are placed into the stepper using a coneshaped cloth harness. Once the mouse has entered the harness, it is secured using a binder clip, which is then magnetically attached to the weight-support system. The legs of the mouse were connected to the robotic arm us-

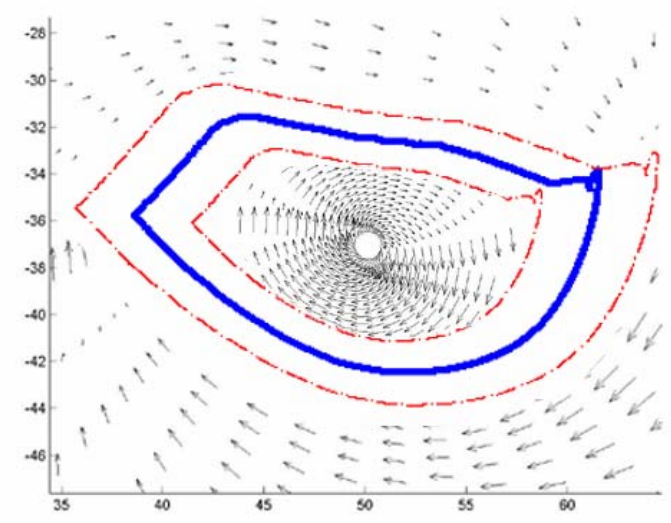

Figure 3: Variability training paradigm I (Band). The desired training trajectory is shown in thick blue. The dotted thin red lines represent the boundaries within which "soft control" is applied to the limbs. The blue arrows correspond to the convergent velocity fields that drive the leg to the band region. (Modified from Cai et al, 2005 [16].) ing a drawstring loop attachment.

Experimental Design: Nine mice were randomly divided into three groups. All three groups received a form of robotic training with quipazine for six weeks. The first group was the control in this experiment and was trained with the fixed robotic training algorithm. The two other experimental groups were each trained with a form of AAN training. Each of the spinal mice groups were trained for $10 \mathrm{~min}$. per day, 5 days per week, for a period of 6 weeks. Measurements of stepping performance were carried out on the $6^{\text {th }}$ day of each training week.

\section{RoBOtIC TRAINING ALgORITHMS}

We implemented three different training algorithms, a repetitive training with a fixed and tightly controlled trajectory and two different types of assist-as-needed (AAN) training algorithms. The two AAN algorithms differed in the amount of interlimb coordination that was imposed during training. The AAN training algorithms were implemented using a velocity field approach where the velocity of the linkage's distal tip is commanded to a specific speed defined by a velocity field. Using the linkage Jacobian matrix, the distal velocities are converted to desired motor velocities.

\section{A. Fixed Robotic Training:}

In this training method, a PID controller performs continuous tracking of a pre-determined pattern. When attached to the mice, the robotic arms actively move the ankle points of the limbs along this fixed trajectory. The imposed $\mathrm{x}$ and $\mathrm{y}$ trajectory of each hindlimb was obtained from a neonatally transected mouse that stepped well. Neonatally transected mice can spontaneously recover functional stepping without pharmacological or mechanical assistance. This pattern was recorded from the neonatal mouse when it was approximately the same age as the adult mice used in this study.

\section{B. Robotic AAN Training Algorithm I: "Band"}

This AAN strategy implements two fixed boundaries: an inner bound and an outer bound, forming a "band" surrounding the desired trajectory. When the ankle lies in sagital plane regions inside (outside) the band, an outward spiraling (inward-spiraling) converging velocity field drives the ankle to the band region. When the ankle leaves the band, the convergent velocity fields will rapidly move it back into the band region (Fig. 3.). Within the band, the ankle is guided by a small constant velocity field tangent to the desired trajectory, i.e. the robot nudges the ankle along the trajectory at a constant speed, but does not enforce specific timing of leg movement along the trajectory (Note, the velocity fields inside the band are omitted in Fig. 3 for clarity). In this way, the animal largely dictates its own motions inside the 


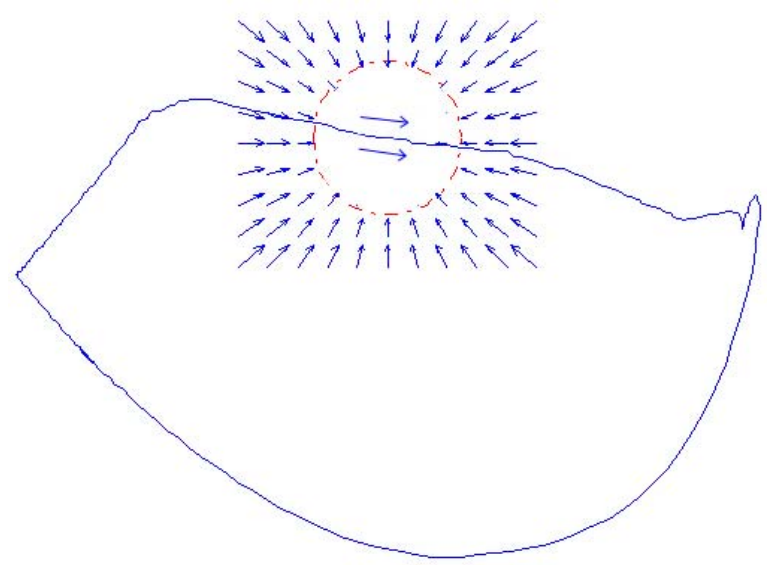

Figure 4: Variability training paradigm II (Window). The desired training trajectory is in blue and the moving window is highlighted in red. The blue arrows correspond to the radial force.

band, with a small bias provided by the robot. Note that this particular instantiation of the AAN paradigm does not impose an interlimb coordination constraint. The computational procedure to construct the velocity field for a given stepping trajectory is outlined in Appendix A.

\section{Robotic AAN Training Algorithm II: "Window."}

The third group received an AAN training paradigm analogous to the second group, but based on a moving "window" geometry. In this approach, a circular "window" moves along the desired trajectory (Fig. 4). The window size was chosen to be $5 \mathrm{~mm}$ in diameter, and was fixed throughout the experiment. We chose this window size because, from observation, it was close to the typical variation of a normal stepping animal. Within the window, a small constant velocity field tangent to the desired trajectory biases the robot's motion, but does not enforce specific timing inside window. Outside the boundary, the robotic movement is guided by a radial force field that points inward with a magnitude proportional to the distance from the circle center: $v=k(d-r)$, where $v$ is the velocity field magnitude, $d$ is the distance between the ankle point and the center of the moving window, $r$ is the window radius, and $k$ is a constant. Hence, when the animal's ankle deviates from the window, it is quickly returned to the window. Within the window, the ankle is gently guided in the direction of the trajectory, thus providing loose timing control. The same strategy was used on both hindlimbs, and the control systems for each leg was timed to provide alternating phasing that is consistent with weight bearing stepping.

\section{DATA ANALYSIS AND EVALUATION METHODS}

We assess the quality of a subject's training in terms of: 1) the number of steps taken by a subject; 2) the periodicity of the subjects steps (i.e., the ability to maintain a regular stepping frequency); and 3) the regularity of the subject's stepping patterns. The following analyses were applied to the data obtained during the weekly tests of each subject (where the robotic trainer is used in its passive mode).

\section{Number of Steps:}

Video footage and plots of ankle position data were used to identify and count the number of steps performed by each mouse. Steps were identified based upon predetermined criteria for step length, height, duration, and degree of interlimb coordination. On each testing day, the 12-sec stepping interval containing the most steps was recorded for subsequent analyses. Both plantar and dorsal steps were accepted. Better subjects performed primarily plantar steps, whereas poorer performing subjects exhibited dorsal steps and paw drag. Immediately after the transection surgery, none of the mice were able to execute any steps. To varying degrees, all of the mice improved their number of steps as a result of training.

\section{Step Periodicity:}

We used the Fast Fourier Transform (FFT), applied to the horizontal component of the stepping trajectories, to quantify step periodicity. Mice that stepped rhythmically exhibited a sharp and distinct fundamental peak in the FFT of their ankle trajectories. The location of the predominant peak corresponds to the most common stepping frequency. Conversely, mice with poor periodicity exhibited either a very broad fundamental peak in the FFT spectrum or, in extreme cases, failed to demonstrate a fundamental peak. To quantify these observations, we measured the full width at half maximum (FWHM) of the fundamental peak. A low FWHM value corresponds to temporally consistent, rhythmic stepping, whereas high measurements typically indicate erratic stepping consistent with stumbling and foot dragging.

\section{Spatial Consistency:}

In addition to the temporal information provided by FFT analysis, we also sought to study changes in spatial stepping consistency throughout the study. We suggest that locomotor improvement can be characterized by more consistent repetition of a nominal trajectory. To measure this quantitatively, we used Principle Components Analysis (PCA). First, we separated the stepping trajectories into their horizontal (or X) and vertical (or Y) components. Next we resampled all the component trajectories so that each test trajectory data set consists of the same number of data points. In this way, only spatial information on the stepping cycles is retained. Then the data are arranged in a matrix form with each columns corresponds to the data points for each $\mathrm{X}$ trajectories of a step and the matrix will have the same number of columns as steps performed with in the ten second interval. For example, given $\mathrm{n}$ steps with $\mathrm{m}$ data points in each, the X-trajectory data matrix will take the following form (with an analogous matrix for the Y-trajectory data). 


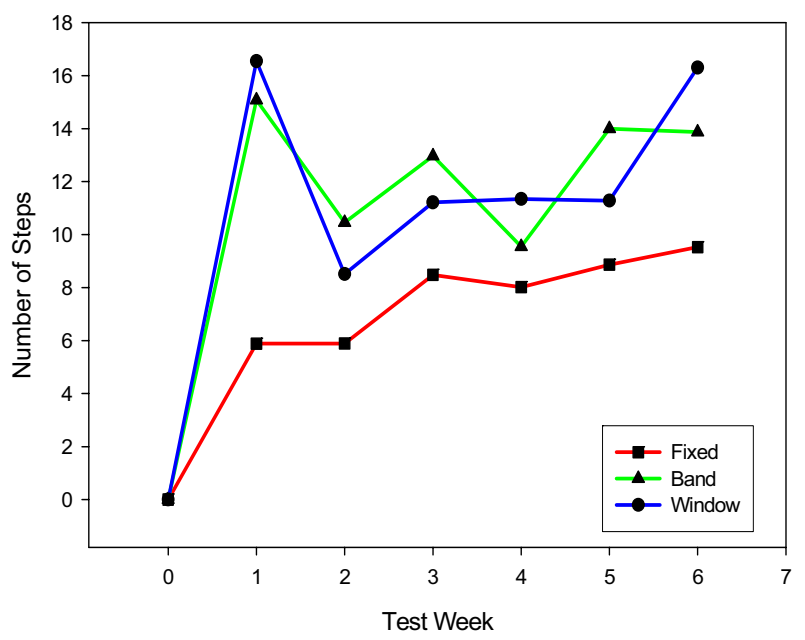

Figure 5: Number of steps performed in $15 \mathrm{sec}$. interval.

$\boldsymbol{X}$ component of the steps

$$
\left[\begin{array}{ccccc}
x_{1,1} & x_{1,2} & \cdots & \cdots & x_{1, n} \\
x_{2,1} & x_{2,2} & & & x_{2, n} \\
\vdots & & \ddots & & \vdots \\
\vdots & & & \ddots & \vdots \\
x_{1, m} & x_{2, m} & \cdots & \cdots & x_{n, m}
\end{array}\right]
$$

We computed the principal components (PC) of the data set, as well as the percentage of the total variance in the data set that is captured by the first principal component. The first principal component can be interpreted as the "nominal" trajectory from the test data set, and the PCA percentage score measures how repeated are the trajectories. A high PCA percentage score corresponds to a subject that consistently executes the same stepping trajectory. A PCA percentage of $45 \%$ was assigned to all mice that could not step on test day, since this is the lowest PCA score that we have ever encountered.

\section{EXPERIMENTAL RESULTS}

The average total number of steps performed in a best 12 second interval by the animals in each group is shown in Fig. 5. The "window" and "band" groups performed more steps than the fixed group. However, there does not seem to be any statistical difference between the two AAN training groups.

In Fig. 6, the inverse of the FWHM is plotted to emphasize the improvement in step rhythm throughout the study. The "window" and "band" AAN training groups consistently stepped more rhythmically than the fixed training group. Although the two variable groups could not be statistically distinguished, the "window" group seemed to recover faster. A score of zero was assigned to all mice that could not step on the first test day.
The PCA percentage score, which measures the spatial regularity of stepping, is plotted in Fig. 7. Although the "window" training group appeared to recover faster, there was no indication of a difference between the step shape consistencies of the three groups. This may be due to the small number of subjects. Alternatively, this supports previous findings that quipazine, rather than the specific training pattern, is primarily responsible for shape consistency [13]. Note that training of any form, combined with quipazine, substantially improves step regularity.

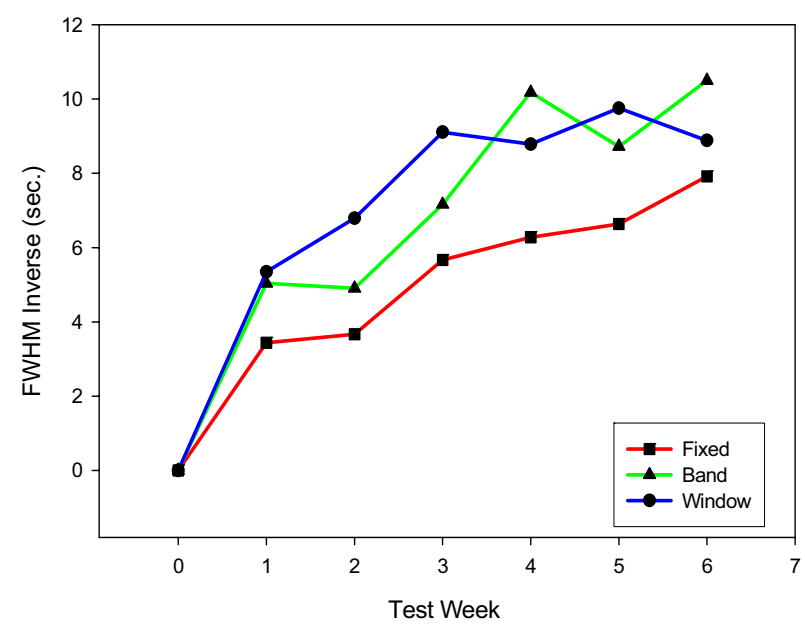

Figure 6: The inverse of FWHM is plotted to emphasize the locomotor improvement over time.

\section{DISCUSSION AND CONCLUSIONS}

A key objective of this study was to ascertain whether providing variability in training enhances stepping recovery. A significant concern is that fixed trajectory training strategies may drive the spinal cord into a state of "learned helplessness," [7, 10] whereby the spinal cord, not challenged to explore potential stepping patterns on its own, will defer to the trainer and give up on learning. No statistically significant differences were observed between the variable "band" and "window" training groups. This may be due to the small $\mathrm{n}$ used. Thus we repeated the experiment with nine animals in each group. Preliminary results suggest that the "window" training paradigm significantly improve the stepping ability of the animal (in number of steps and step periodicity) compares to animals from the "band" and "fixed".

Another observation from this study was that interlimb coordination must be controlled in order to maximize locomotor recovery. Although it had been shown that spinal cats can adapted to different walking speeds on a split treadmill [11], we found interlimb coordination plays an important role in training adult spinal mice to step. In the pilot study, mice trained with the "band" pattern still improved in their stepping performance, but 


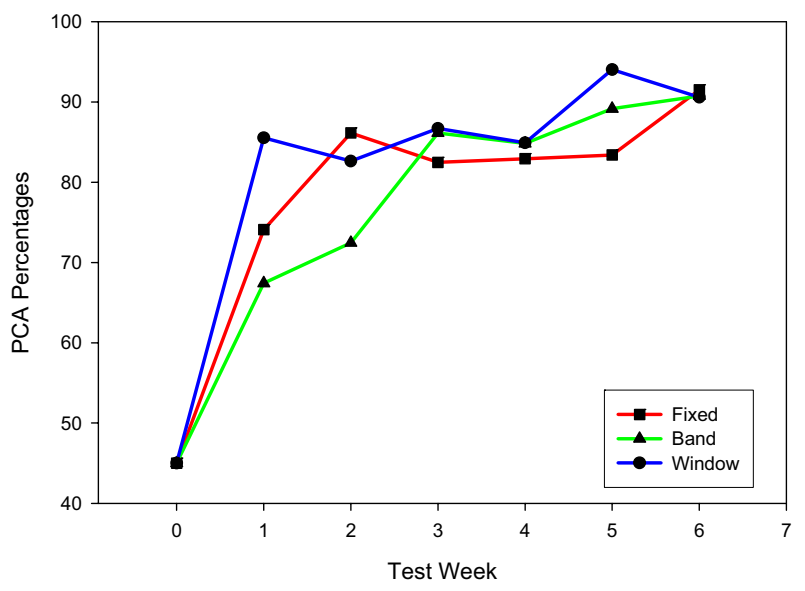

Figure 7: The PCA scores throughout training.

their steps were typically arrhythmic and frequently interrupted by dragging. In contrast, stepping executed by the "fixed" and "window" trained groups were rhythmic and prolonged. This was observed when we look at the location of the FFT peaks, which showed that stepping in the "window" and "fixed" groups converged to a frequency near $1 \mathrm{~Hz}$ as the study progressed, a result consistent with constant speed treadmill locomotion at $3 \mathrm{~cm} / \mathrm{sec}$. These differences became even more evident in the full-scale study. Preliminary results demonstrated significant differences between the "window" group and the "band" group, where the "band" group performed similar to the "fixed" group in all three measurements.

Principal components analysis did not elucidate a difference in the shape consistency of the three groups. By week 3, the average PCA score of all three groups was greater than $80 \%$, indicating that all of the mice could step rhythmically. This result supports previous conclusions that quipazine rather than step training has the larger influence on step shape consistency [12]. Preliminary results from the full-scale study showed similar end effect.

Sensory information is critical to motor learning [13, 14]. The pattern and timing of assistance provided during step training dictates the extent to which the injured spinal cord can recover motor function. The results suggests that failure to enforce contralateral phasing of the legs during training limits stepping recovery compared to mice trained with an alternating gait. It is likely that the spinal cord is similarly highly sensitive to other proprioceptive cues. As demonstrated here, poorly conceived or poorly implemented training algorithms may actually inhibit stepping recovery.

This emphasizes a fundamental advantage of using robotic devices that can consistently and accurately apply defined locomotor training paradigms while simultaneously maintaining a thorough quantitative record of both training and performance parameters. Used with data optimization schemes, quantitative data are teaching us which parameters to focus on when devising novel train- ing strategies. By developing our understanding of how sensory inputs affect spinal learning, we can "tune the spinal cord to learn."

\section{ACKNOLEDGEMENT}

The author will like to thank Dr. Roland Roy and Veronica Zhong for performing all the necessary surgery, Dr. Jorge G. Cham for his helpful advices, and Maynor Herrera for all the animal care.

\section{REFERENCES}

[1] S. Hesse, H. Schmidt, C. Werner, and A. Bardeleben, "Upper and lower extremity robotic devices for rehabilitation and for studying motor control," Curr Opin Neurol, vol. 16, pp. 705-10, 2003.

[2] R. D. de Leon, M. D. Kubasak, P. E. Phelps, W. K. Timoszyk, D. J. Reinkensmeyer, R. R. Roy, and V. R. Edgerton, "Using robotics to teach the spinal cord to walk," Brain Res Brain Res Rev, vol. 40, pp. 267-73, 2002.

[3] D. J. Reinkensmeyer, J. L. Emken, and S. C. Cramer, "Robotics, motor learning, and neurologic recovery," Annu Rev Biomed Eng, vol. 6 , pp. 497-525, 2004

[4] S. E. Fasoli, H. I. Krebs, and N. Hogan, "Robotic technology and stroke rehabilitation: translating research into practice," Top Stroke Rehabil, vol. 11, pp. 11-9, 2004.

[5] H. Barbeau and S. Rossignol, "Initiation and modulation of the locomotor pattern in the adult chronic spinal cat by noradrenergic serotonergic and dopaminergic drugs," Brain Res, vol. 546, pp. 250-60, 1991.

[6] M. R. Brumley and S. R. Robinson, "The serotonergic agonists quipazine, CGS-12066A, and alpha-methylserotonin alter motor activity and induce hindlimb stepping in the intact and spinal rat fetus," Behav Neurosci, vol. 119, pp. 821-33, 2005.

[7] E. S. Landry and P. A. Guertin, "Differential effects of 5-HT1 and 5-HT2 receptor agonists on hindlimb movements in paraplegic mice," Prog Neuropsychopharmacol Biol Psychiatry, vol. 28, pp 1053-60, 2004

[8] A. J. Fong, L. L. Cai, C. K. Otoshi, D. J. Reinkensmeyer, J. W. Burdick, R. R. Roy, and V. R. Edgerton, "Spinal cord-transected mice learn to step in response to quipazine treatment and robotic training," J Neurosci, vol. 25, pp. 11738-47, 2005

[9] J. M. Hausdorff, "Gait variability: methods, modeling and meaning," J Neuroengineering Rehabil, vol. 2, pp. 19, 2005.

[10] N. F. Skinner, "Learned helplessness: performance as a function of task significance," J Psychol, vol. 102, pp. 77-82, 1979.

[11] R. N. Wool, D. Siegel, and P. R. Fine, "Task performance in spinal cord injury: effect of helplessness training," Arch Phys Med Rehabil, vol. 61, pp. 321-5, 1980.

[12] M. Wirz, R. Hornby, R. Rupp, and V. Dietz, "Locomotor training with a driven gait orthosis in incomplete spinal cord injury," Gait Posture, vol. Volume 21, pp. S74, 2005.

[13] D. Orsal, J. Y. Barthe, M. Antri, D. Feraboli-Lohnherr, A. Yakovleff, M. Gimenez y Ribotta, A. Privat, J. Provencher, and S. Rossignol, "Locomotor recovery in chronic spinal rat: long-term pharmacological treatment or transplantation of embryonic neurons?," Prog Brain Res, vol. 137, pp. 213-30, 2002.

[14] J. W. Grau, D. G. Barstow, and R. L. Joynes, "Instrumental learning within the spinal cord: I. Behavioral properties," Behav Neurosci, vol. 112, pp. 1366-86, 1998.

[15] A. J. Fong, V. R. Edgerton, L. L. Cai, C. K. Otoshi, W. K. Timoszyk, M. Merlo, A. J. Bigbee, H. Zhong, R. R. Roy, D. J. Reinkensmeyer, and J. W. Burdick, "Effects of quipazine and robotic training on spinal mice, pp.," Washington, DC: Soc Neurosci Abstr, vol. Program No. 498.20, 2003.

[16] A. L. Behrman and S. J. Harkema, "Locomotor training after human spinal cord injury: a series of case studies," Phys Ther, vol. 80 , pp. 688-700, 2000.

[17] V. Dietz, R. Muller, and G. Colombo, "Locomotor activity in spinal man: significance of afferent input from joint and load receptors," Brain, vol. 125, pp. 2626-34, 2002. 